\title{
Application of Colorimetric Solid Phase Extraction (C-SPE) to Monitoring Nickel(II) and Lead(II) in Spacecraft Water Supplies
}

\author{
Neil C. Dias, Daniel B. Gazda, James S. Fritz, and Marc D. Porter \\ Ames Laboratory - U.S.D.O.E. \\ Department of Chemistry, lowa State University \\ Ames, lowa, USA. \\ Jeff Rutz, Paul Mudgett, and John Schultz \\ Wyle Laboratories \\ Houston, Texas, USA.
}

Copyright (C) 2004 SAE International

\begin{abstract}
Archived water samples collected on the International Space Station (ISS) and returned to Earth for analysis have, in a few instances, contained trace levels of heavy metals. Building on our previous advances using Colorimetric Solid Phase Extraction (C-SPE) as a biocide monitoring technique [1-3], we are devising methods for the low level monitoring of nickel(II), lead(II) and other heavy metals. C-SPE is a sorption-spectrophotometric platform based on the extraction of analytes onto a membrane impregnated with a colorimetric reagent that are then quantified on the surface of the membrane using a diffuse reflectance spectrophotometer. Along these lines, we have determined nickel(II) via complexation with dimethylglyoxime (DMG) and begun to examine the analysis of lead(II) by its reaction with 2,5 dimercapto-1,3,4-thiadiazole (DMTD) and 4-(2pyridylazo)-resorcinol (PAR). These developments are also extending a new variant of C-SPE in which immobilized reagents are being incorporated into this methodology in order to optimize sample reaction conditions and to introduce the colorimetric reagent. This paper describes the status of our development of these two new methods.
\end{abstract}

\section{INTRODUCTION}

Water samples collected on the International Space Station (ISS) and analyzed upon return to Earth have in a few instances contained traces of lead, cadmium, and nickel [4]. These contaminants have been identified as the result of the aging and degradation of compartments in the water distribution and cooling systems. Although many of the sources of these metals have been isolated and corrected, these contaminant breakthroughs strongly underscore the potential for a serious compromise in ISS water quality as the many on-board systems wear and age. A simple, reliable analytical method is therefore needed for the onboard monitoring of lead(II), nickel(II), and other heavy metals in spacecraft water supplies. Moreover, such a method must be selective for the target analyte (i.e., minimal interferences from other contaminants), have sufficient sensitivity to detect contaminants at low concentrations (e.g., the current allowable limits of lead and nickel in spacecraft water are 50.0 and $30.0 \mathrm{ppb}$, respectively) [3], and be capable of operation in microgravity. This paper describes our efforts to develop methods based on colorimetric solid phase extraction (C-SPE) that meet the onboard monitoring needs of the space program for both nickel(II) and lead(II).

As described previously $[1,2,5], \mathrm{C}-\mathrm{SPE}$ integrates an effective preconcentration step with the measurement of a colored analyte complex by diffuse reflectance spectroscopy on the surface of a small extraction disk. In this method, a membrane disk that is loaded with polymeric extractive particles is mounted in a filter holder after impregnation with a colorimetric reagent. A syringe containing a known volume of sample is then attached to the holder and the sample is passed through the disk, forming a colored analyte-reagent complex on the disk. The amount of analyte is determined on the disk surface using a small, hand-held diffuse reflectance spectrophotometer. Quantization of the analyte is accomplished by means of a calibration plot of the Kulbelka-Munk function at a single wavelength, $F(R)$, against analyte concentration. The complete procedure requires only 1-2 min. Furthermore, this methodology uses simple, lightweight equipment, and is capable of operation in microgravity $[6,7]$.

Our previous work developed C-SPE methods for determination of the biocides $\mathrm{I}_{2}$ and $\mathrm{Ag}(1)$ and heavy metals nickel(II), chromium(VI), copper(II), and iron(III) $[1,2,5,8]$. We have also described techniques that can be used to mix reagents with the sample and to manipulate the microscopic environment of the membrane by impregnation with surfactants and/or other 
chemical modifiers $[2,5]$. Furthermore, we have tested the performance of these methods in microgravity via $\mathrm{KC}-135$ flights $[6,7,9]$, and have recently shown a strong correlation between flight and ground-based data for iodine and silver $(I)[10]$. The work herein summarizes the status of our efforts to create and/or refine methods for the determination of nickel(II) and lead(II). This report focuses particularly on examining various colorimetric reagents for these determinations as well as the use of additives to manipulate the membrane microenvironment in order to effectively enhance the extraction process and/or improve the rate of color development during complexation. We first describe our latest results in the development of a C-SPE method for nickel(II), which is then followed by a summary of our efforts to design a CSPE method for lead(II).

\section{EXPERIMENTAL}

REAGENTS AND CHEMICALS - All solutions were prepared daily with deionized water that was further purified by a Millipore Milli-Q water purification system.

Standard lead(II) solutions - A $100.0 \mathrm{~mL}$ volume of stock solution (100.0 ppm) of lead(II) was prepared by dissolving an appropriate amount of lead nitrate (Aldrich) in deionized water. This stock was stabilized against hydrolysis by the addition of $1.0 \mathrm{~mL}$ of $\mathrm{HNO}_{3}$ (dilute). More dilute lead(II) solutions were prepared by further dilutions of aliquots of the stock solution with deionized water.

PAR solution - A $0.05 \%$ PAR solution was prepared by dissolving $50.0 \mathrm{mg}$ of the monosodium salt of this reagent (Aldrich) in $100.0 \mathrm{~mL}$ of deionized water. The $\mathrm{pH}$ was adjusted to 9.0-10.0 using a dilute $\mathrm{NH}_{4} \mathrm{OH}$ solution.

KI solution - A $100.0 \mathrm{~mL}$ volume of a solution of $\mathrm{I}^{-}(0.2 \mathrm{M})$ was prepared by dissolving $3.32 \mathrm{~g}$ of $\mathrm{KI}$ (Aldrich) in deionized water.

Cetylpyridinium chloride (CPC) solution - A 3.0\% solution of CPC was prepared by dissolving $3.0 \mathrm{~g}$ of the compound (Aldrich) in $100.0 \mathrm{~mL}$ of deionized water.

DMDT solution - This solution ( $0.05 \mathrm{M})$ was prepared by dissolving the appropriate amount of 2,5-dimercapto1,3,4-thiadiazole, dipotassium salt (Aldrich) in deionized water.

Tetrabutylammonium chloride - A $100.0 \mathrm{~mL}$ stock solution $(0.5 \mathrm{M})$ of tetrabutylammonium hydroxide (Aldrich) was prepared by dissolving the requisite amount of this reagent in deionized water. More dilute solutions of the reagent were prepared as required.

Standard nickel(II) solutions - Solutions with nickel(II) concentrations of $0.50,1.0,2.0$, and $5.0 \mathrm{ppm}$ were prepared in Nalgene bottles by mixing the appropriate mass of a nickel(II) atomic absorption standard (1000.0 ppm, Spex Certiprep, Metuchen, NJ, USA) with either deionized water or borax buffer (described below) and bringing the solution to a final mass of $30.0 \mathrm{~g}$

Buffer solutions - Buffer solutions with pH values of 2.0, $5.0,7.0,8.0,9.0$, and 10.0 were prepared to determine optimum reaction conditions for nickel(II). The $\mathrm{pH}$ of these buffers was determined using an Orion model $520 \mathrm{~A} \mathrm{pH}$ meter. The pH 2.0 buffer consisted of $0.05 \mathrm{M}$ potassium chloride adjusted to the correct $\mathrm{pH}$ with $0.1 \mathrm{M}$ $\mathrm{HCl}$. The $\mathrm{pH} 5.0$ buffer was prepared from $0.05 \mathrm{M}$ potassium hydrogen phthalate adjusted to $\mathrm{pH} 5.0$ with $0.1 \mathrm{M} \mathrm{NaOH}$. The $\mathrm{pH} 7.0$ buffer was prepared from $0.05 \mathrm{M}$ potassium dihydrogen phosphate that was brought to the correct $\mathrm{pH}$ with $0.1 \mathrm{M} \mathrm{NaOH}$. The $\mathrm{pH} 8.0$, 9.0 , and 10.0 buffers were all made by adjusting the $\mathrm{pH}$ of a $0.013 \mathrm{M}$ borax solution with either $0.1 \mathrm{M} \mathrm{HCl}$ or $0.1 \mathrm{M} \mathrm{NaOH}$.

Buffer wool - A buffer slurry was prepared by adding $2.0 \mathrm{~g}$ of sodium tetraborate decahydrate to $20.0 \mathrm{~mL}$ of deionized water. This solution was shaken to suspend any undissolved salt and poured over a $100-\mathrm{mm}$ diameter disk of glass wool (Fischer) in a polystyrene Petri dish. The dish was heated in an oven at $50^{\circ} \mathrm{C}$ overnight to evaporate the solvent and therefore entrap the salt within the wool.

DMG chads - A $0.022 \mathrm{M}$ solution of DMG (Aldrich) was prepared by dissolving $0.25 \mathrm{~g}$ of DMG in $100.0 \mathrm{~mL}$ of methanol. Whatman no. 1 qualitative filter paper was cut into $10-\mathrm{mm}$ chads. Each chad was then treated with $20.0 \mu \mathrm{L}$ of the DMG solution and allowed to air dry.

C-SPE disks for nickel(II) - Empore ${ }^{T M}$ SDB-XC $47 \mathrm{~mm}$ extraction membranes (3M) were used as a matrix for collection of the colorimetric complex. Membranes were used as received after being cut into $13-\mathrm{mm}$ disks in order to fit into a polypropylene filter holder.

CPC-impregnated membranes. - The membranes were prepared by impregnating Empore ${ }^{\mathrm{TM}}$ SDB-XC extraction membranes with CPC. This process was carried out by mounting a membrane on a Millipore $47-\mathrm{mm}$ glass vacuum filter holder and pulling $10.0 \mathrm{~mL}$ of the impregnating reagent solution (CPC) through by vacuum. Residual solution was removed by vacuum for an additional $1-2 \mathrm{~min}$. The membranes were then cut into 13-mm disks, and air dried for 1-2 $\mathrm{h}$.

INSTRUMENTATION - A BYK-Gardner color-guide sphere $\left(\mathrm{d} / 8^{\circ}\right)$ diffuse reflectance spectrophotometer (DRS) (Model LCB-6830) was used to collect the spectral data from the impregnated disks. This hand-held spectrophotometer is small, lightweight, battery operated, and can collect reflectance data over the entire visible spectral range $(400-700 \mathrm{~nm})$ at a $20-\mathrm{nm}$ resolution in only $1.5 \mathrm{~s}$. The resulting spectrum is shown on the instrument display panel or can be downloaded to a computer. The aperture of the instrument enables reflectance readings to be made on a $13-\mathrm{mm}$ diameter disk, which mates to the spectrophotometer through a sample positioner. 
SOFTWARE - The DRS was interfaced to a computer by a serial cable. Spectra were then transferred to the computer and downloaded using a modified version of BYK-Gardner QC-Link software. The data were then transferred to another worksheet to calculate KubelkaMunk functions and plot calibration curves. The KubelkaMunk function, $(F(R))[11]$, is

$F(R)=(1-R)^{2} / 2 R$

where $R$ is the absolute diffuse reflectance. We note that $F(R)$ is directly related to the concentration of the complex $C$ by

$F(R)=2.303 \approx \mathrm{C} / \mathrm{s}$

where $\varepsilon$ is the absorbtivity of the sample and $s$ is the scattering coefficient of the sample surface.

EXTRACTION PROCEDURE AND READOUT FOR LEAD(II) AND NICKEL(II) - Plastic syringes of differing volumes $(1.0$ and $10.0 \mathrm{~mL})$ were employed to meter known amounts of sample through the $13-\mathrm{mm}$ diameter disks. Each disk, after impregnation or as received, was placed on the support screen on the bottom of a plastic, threaded filter holder (Swinnex Filter Holders; Fisher No. 09-753-10ASX00 0013 00), which was then tightly screwed into the top of the holder. The syringe was connected to the holder via a Luer Lock fitting, and the sample was passed manually through the disk; the extraction step required $\sim 0.5 \mathrm{~min}$. After extraction, the holder was separated from the syringe and the sample disk was dried by the passage of $60.0 \mathrm{~mL}$ of air. The sample holder was then opened and the lower portion was mounted on the spectrophotometer to acquire the spectrum.

\section{RESULTS AND DISCUSSION}

\section{NICKEL(II) METHODOLOGY}

Methodology Development - Experiments aimed at exploring C-SPE methods for nickel(II) have focused on dioximes as colorimetric reagents and are summarized in our earlier reports [5,8]. Many different nickel(II)dioxime complexes are retained on untreated Empore ${ }^{\mathrm{TM}}$ disks, but the reaction of the metal ion with the impregnated reagents was unacceptably slow. One parameter that can potentially be manipulated to accelerate the reaction rate is $\mathrm{pH}$. Dioximes couple in a $2: 1$ stoichiometry with nickel(II) by the loss of one proton from each of the two bound chelates to form a neutrally charged complex. It is therefore possible that by buffering the sample solution at a $\mathrm{pH}$ greater than the $\mathrm{pK}_{\mathrm{a}}$ of the colorimetric reagent, the rate of color development on the membrane surface will proceed much faster.

In testing this strategy over a $\mathrm{pH}$ range of 2-10, we found that buffering at $\mathrm{pH} 9.0$ yielded the fastest color development. These experiments also revealed that sample volumes greater than $1.0 \mathrm{~mL}$ did not appreciably increase the analyte signal. Accordingly, $1.0 \mathrm{~mL}$ samples were used in all subsequent studies.

The $F(R)$ responses at $540 \mathrm{~nm}$, the maximum of the Kubelka-Munk function for the membrane-captured precipitate, are plotted against sample concentration (pH 9.0) in Figure 1. The plot exhibits a linear dependence up to $5.0 \mathrm{ppm}$. The lower limit of detection, extrapolated from a regression analysis of the data and the blank response at three times its standard deviation, was $0.47 \mathrm{ppm}$. Importantly, this limit corresponds to a mass detection of $\sim 500 \mathrm{ng}$ in a $1.0 \mathrm{~mL}$ sample and requires only $\sim 40 \mathrm{~s}$ for full sample work up and analysis.

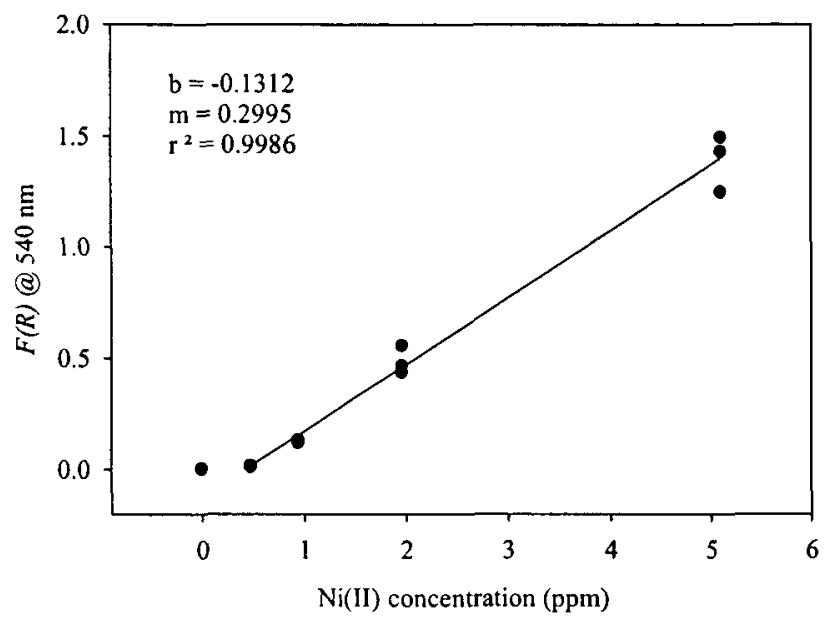

Figure 1. Calibration curve for nickel(II) in buffered sample solutions $(\mathrm{pH} 9.0)$.

Samples prepared with the same concentrations as the buffer samples in deionized water were employed to examine the use of an immobilized buffer (i.e., the buffer wool described in the Experimental section) for $\mathrm{pH}$ adjustment. Without buffer treatment, there is no detectable color formation on the membrane. When the buffer wool is used to adjust the sample $\mathrm{pH}$, the results in Figure 2 show excellent agreement with those obtained using the buffered samples. The buffer cartridge was used in two configurations: 1) when integrated as part of reagent chad/collection membrane assembly, and 2) when collecting the sample in the syringe, but before attachment of the reagent chad assembly. Both configurations performed well, but the volume of the buffer cartridge $(\sim 0.4 \mathrm{~mL})$ increased the total dead volume of the assembly to $1.2 \mathrm{~mL}$, making it difficult to reliably pass a $1.0 \mathrm{~mL}$ sample through the entire assembly.

As a consequence of this difficulty, the buffer wool was used in-line during syringe filling in all experiments. Comparisons of the slope and intercept values calculated from the buffered samples (Figure 1) and the deionized water samples passed through the buffer wool (Figure 2) yielded differences of only 2.5 and $0.91 \%$, respectively, demonstrating the ability to reliably manipulate the solution $\mathrm{pH}$ with the buffer wool. 


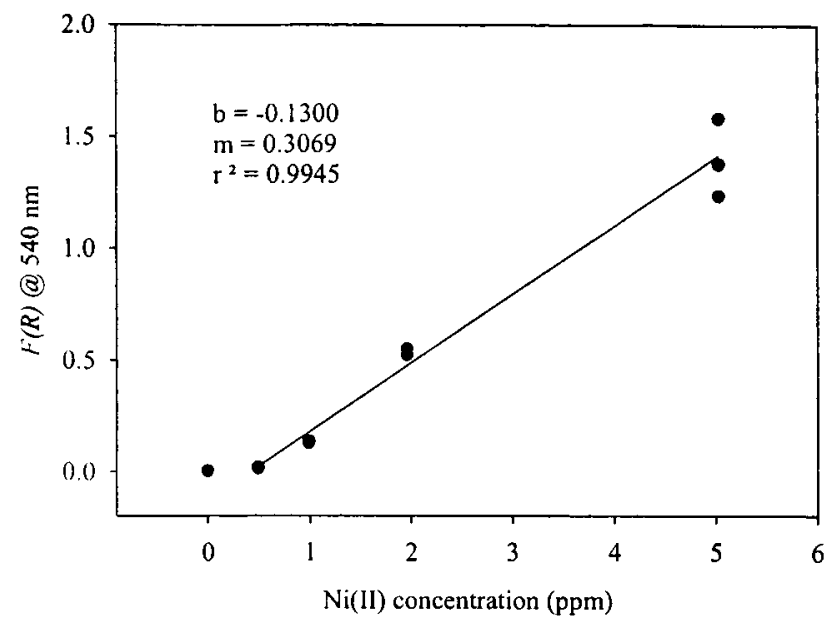

Figure 2. Calibration curve for nickel(II) samples prepared in deionized water and collected through buffer wool.

Interference Testing - There are several metal ions that interfere with the formation of the nickel(II)- $-\mathrm{DMG}_{2}$ complex, including iron(III), manganese(II), cobalt(II), and copper(II). To determine the extent of interference from each species, solutions containing $1.0 \mathrm{ppm}$ of iron(III), manganese(II), copper(II), or cobalt(II) were prepared from their corresponding sulfate salts. Of the species tested, none produced signals that could be assigned to in-absentia nickel(II). The iron(III) solution yielded a yellow membrane, probably from the precipitation of iron(III) hydroxide at pH $9.0\left(\mathrm{~K}_{\mathrm{sp}}=2.64 \times 10^{\circ}\right.$ $\left.{ }^{39}\right)$.

Solutions containing equal concentrations $(1.0 \mathrm{ppm})$ of the interfering metal ion and nickel(II) and a 10-fold excess of interfering metal ion were also tested. Manganese(II), copper(II), and cobalt(II) showed no interference to the determination of nickel(II) when present at an equal concentration. The presence of iron(III), however, caused a positive deviation in the signal. When the solutions with the 10-fold excess of interferant were analyzed, all species except manganese(II) interfered with the nickel(II) determination. Copper(II), cobalt(II), and iron(III) all tended to yellow the surface of the membrane.

These results, which are summarized in Table 1 are promising. Three of the four metal ions tested, copper(II), cobalt(II), and manganese(II), do not interfere when present at comparable concentrations to nickel(II). Moreover, copper(II) and manganese(II) are analytes mandated for ISS water quality monitoring, and are likely to be present in samples used for nickel(II) quantification. Also, we believe that since of the interferences cause a yellowing of the membrane surface, it may be possible to simultaneously monitor for these species in the presence of nickel(II) by employing multiple detection wavelengths. Work to test this hypothesis is underway. Based on these findings, we are also beginning to develop a flight package for testing the performance of the C-SPE nickel(II) methodology in microgravity.

Table 1. Interference studies using $1: 1$ and $10: 1$ ratios of interferant to nickel(II).

\begin{tabular}{ccccc}
\hline $\begin{array}{c}\text { Metal } \\
(\mathrm{M})\end{array}$ & $\begin{array}{c}\mathrm{M} \\
(\mathrm{mg} / \mathrm{L})\end{array}$ & $\begin{array}{c}\mathrm{Ni}^{2+} \\
(\mathrm{mg} / \mathrm{L})\end{array}$ & $\begin{array}{c}F(R) \\
\text { calculated }\end{array}$ & $\begin{array}{c}F(R) \\
\text { obtained }\end{array}$ \\
\hline $\mathrm{Cu}^{2+}$ & 0.995 & 0.995 & 0.175 & 0.167 \\
$\mathrm{Mn}^{2+}$ & 1.00 & 0.994 & 0.175 & 0.165 \\
$\mathrm{Fe}^{3+}$ & 0.986 & 0.986 & 0.173 & 0.363 \\
$\mathrm{Co}^{2+}$ & 0.997 & 0.990 & 0.174 & 0.173 \\
\hline $\mathrm{Cu}^{2+}$ & 10.00 & 0.980 & 0.170 & 0.072 \\
$\mathrm{Mn}^{2+}$ & 9.86 & 0.980 & 0.170 & 0.197 \\
$\mathrm{Fe}^{3+}$ & 9.63 & 0.944 & 0.160 & 0.010 \\
$\mathrm{Co}^{2+}$ & 10.10 & 0.979 & 0.170 & 0.012 \\
\hline
\end{tabular}

\section{LEAD(II) METHODOLOGY}

The classical methods for the spectrophotometric determination of lead(II) utilize chromogenic chelating reagents such as dithizone, pyridylazoresorcinol (PAR), and 2,5-dimercapto-1,3,4-thiadiazole (DMTD) [12-17]. However, the assay with dithizone requires extraction with an organic phase (e.g., chloroform or carbon tetrachloride), is less sensitive than the methods using PAR and DMTD, and the resulting complex is unstable $[18,19]$. Dithizone is therefore not suitable for deployment on ISS. The methods based on PAR and DMTD, in contrast, can be carried out in aqueous solutions, and yield a more intensely colored and stable complex [14]. This section describes our preliminary results from assessing the use of PAR and DMTD as colorimetric reagents for the determination of lead(II) by C-SPE.

\section{INVESTIGATION OF DMTD}

Methodology Development - DMTD provides reasonable spectrophotometric sensitivity and selectivity for lead(II) in a solution-based assay $[16,20]$. DMTD reacts with lead(II) in weakly acidic solution to form a 2:1 dianionic complex [20], requiring the creation of a cationic extraction medium for use in C-SPE. We have found that such an environment can be prepared by impregnating the membrane with a cationic surfactant like cetylpyridinium chloride (CPC). Accordingly, $1.0 \mathrm{~mL}$ of $0.05 \mathrm{M}$ DMTD was mixed with $10.0 \mathrm{~mL}$ of standard lead(II) solutions (1.0-7.0 ppm) and passed through a membrane impregnated with CPC. Unfortunately, any excess DMTD, which is a strongly hydrophobic anion, is also extracted by the CPC-impregnated membrane, imparting a color to the membrane that interferences with the detection of the DMTD:lead(II) complex.

Formation of an ion pair by the metal ion complex with an organic cation clearly enhances the extraction of the anionic lead(II):DMTD ${ }_{2}$ complex. We therefore postulated that lowering the hydrophobicity of the microenvironment created by impregnation with the 16carbon polymethylene chain of CPC would reduce the 
uptake of excess DMTD. Accordingly, the short chain quaternary ammonium chloride salts, selected to have a lower hydrophobicity, were mixed with the lead(II) sample, along with DMTD, before passage through a non-impregnated Empore ${ }^{T M}$ disk. These results are summarized by the histogram in Figure 3 , which shows the response found both on the top and bottom of the membrane and serves as a rough measure of extraction efficiency and product bleed-through. Tetraethylammonium- and tetrapropylammoniumchloride yielded a minor increase in the amount of lead(II):DMTD ${ }_{2}$ extracted with respect to as-received membrane, but the complex clearly bled through the disk at an unacceptable level. Tetrabutylammonium chloride, on the other hand, gave a significantly higher $F(R)$, and almost no detectable level of bleedthrough. An even higher $F(R)$ was obtained when the concentration of tetrabutylammonium chloride was increased from $0.05 \mathrm{M}$ to $0.5 \mathrm{M}$, but there was no further change when the concentration was increased from $0.5 \mathrm{M}$ to $1.0 \mathrm{M}$. There was also no evidence for the uptake of unreacted DMTD. In view of these findings, all subsequent work was carried out using $0.5 \mathrm{M}$ tetrabutylammonium chloride.

We also found that a moderately acidic $\mathrm{pH}$ was necessary for optimum color development [16]. Acidification was accomplished by adding a fixed amount of a dilute $\mathrm{HCl}$ solution to the sample along with the DMTD. The highest value of $F(R)$ at $400 \mathrm{~nm}$ was obtained by adding $1.0 \mathrm{~mL}$ of $0.05 \mathrm{M} \mathrm{HCl}$ to a $10.0 \mathrm{~mL}$ sample.

The next step analyzed these results in order to construct a calibration curve for the analysis and to determine the limit for lead(II) detection. As such, the following reagents were added to $10.0 \mathrm{~mL}$ samples containing varying concentrations of lead(II): $1.0 \mathrm{~mL}$ of $0.005 \mathrm{M} \mathrm{HCl}, 1.0 \mathrm{~mL}$ of $0.05 \mathrm{M}$ DMTD, and $1.0 \mathrm{~mL}$ of $0.5 \mathrm{M}$ tetrabutylammonium chloride. This solution was then passed through an Empore ${ }^{\mathrm{TM}}$ disk and the diffuse reflectance was measured at $400 \mathrm{~nm}$. After conversion of the diffuse reflectance data to values of $F(R)$, a linear plot was obtained from 0.05 to $1.0 \mathrm{ppm}$, and is presented in Figure 4. A regression analysis yielded: $F(R)=$ $0.909\left[\right.$ lead(II)] -0.017 , with a $R^{2}$ of 0.9923 and a limit of detection of $6.0 \mathrm{ppb}$. Additional experiments revealed that the observed linearity continues up to $-5.0 \mathrm{ppm}$. Based on these results, we opted to carry out a preliminary assessment of interferences.

Interference Testing - The potential interference by other metal ions was studied by comparing the $F(R)$ values of a $0.5 \mathrm{ppm}$ lead(II) sample with a sample that also contained $0.5 \mathrm{ppm}$ or $0.1 \mathrm{ppm}$ of other metal ions (i.e., copper(II), cadmium(II), nickel(II), and zinc(II)). At $0.5 \mathrm{ppm}$, cadmium(II) gave a slightly lower value for $F(R)$, but copper(II), zinc(II) and nickel(II) each gave significantly higher responses. Addition of $0.1 \mathrm{ppm}$ of these metal ions had the following effect on the $F(R)$ for $0.5 \mathrm{ppm}$ of lead(II): copper(II), $+15 \%$; cadmium(II), $-11 \%$; nickel(II), $-4 \%$; and zinc(II), $-8 \%$. While the findings for nickel(II) and zinc(II) are acceptable, the level of interferences by copper(II) and cadmium(II) are more problematic. This situation led us to begin an assessment of the use of a masking agent to enhance selectivity.

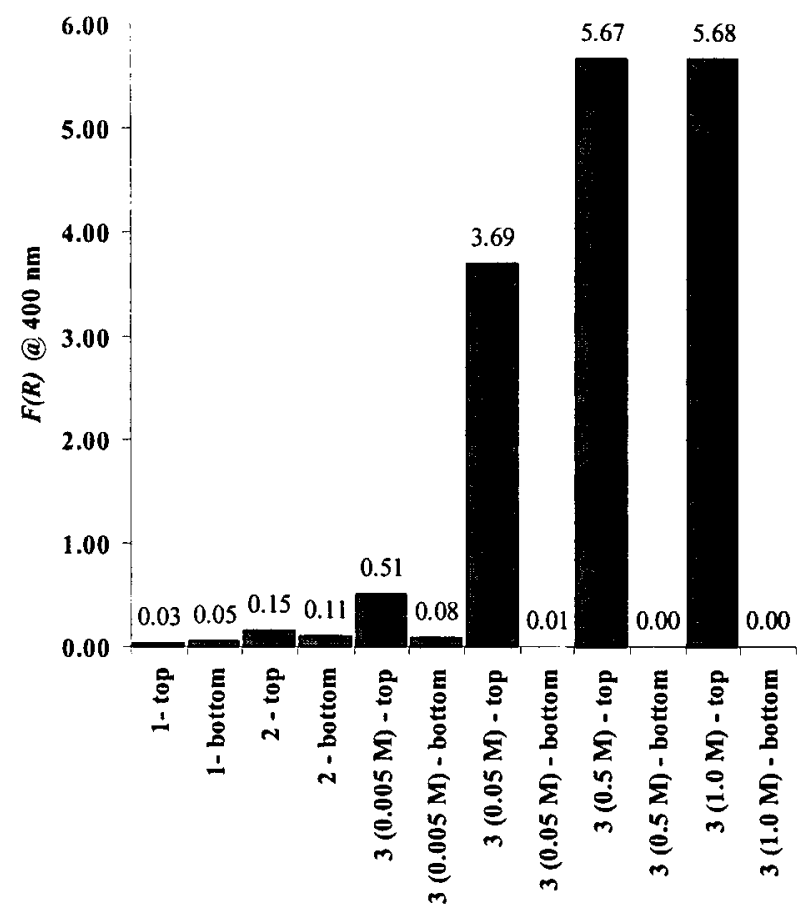

Figure 3. Effect of a quaternary ammonium salt on the Kubelka-Munk factor for lead(II) at $400 \mathrm{~nm}$ : (1) tetraethyl ammonium chloride $(0.005 \mathrm{M})$; $\quad$ (2) tetrapropyl ammonium chloride $(0.005 \mathrm{M})$, and (3) tetrabutyl ammonium hydroxide $(0.005 \mathrm{M}, 0.05 \mathrm{M}$, and $1.0 \mathrm{M})$

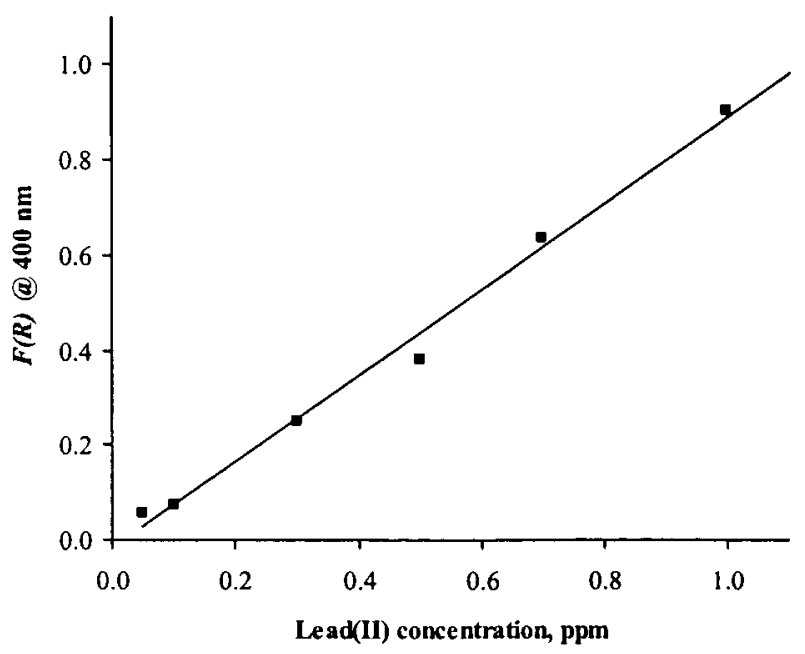

Figure 4. Calibration plot for lead(II):DMTD ${ }_{2}$ complex.

Tartrate was investigated as a possible masking reagent because it forms soluble, colorless complexes with a number of metal ions, and has proven effective in methodologies based on classical spectrophotometry [16]. Addition of tartrate, as well as DMTD and 
tetrabutylammonium chloride to the lead(II) standard, increased $F(R)$ for $0.5 \mathrm{ppm}$ of lead(II) by $\sim 11 \%$. However, addition of tartrate to a sample containing $0.5 \mathrm{ppm}$ lead(II) and $0.1 \mathrm{ppm}$ of another metal ion increased $F(R)$ by $70 \%$ for cadmium(II) and $21 \%$ for copper(II). In contrast, $F(R)$ was virtually unchanged when tartrate was added to lead solutions containing zinc(II) or nickel(II). These studies indicate that tartrate is not effective as a masking agent when employed in CSPE methodology, which strongly contradicts its performance in the solution-based spectrophotometric determinations of lead(II) [16]. Experiments are presently being designed to ascertain the basis of this reactivity difference, as well as to test the utility of other masking agents (e.g., citrate and thiocyanate).

Investigation of PAR - Although PAR reacts with many other metal ions (e.g., copper(II), cadmium(II), bismuth(II), nickel(II), cobalt(II), and iron(III)), its selectivity for lead(II) can be markedly enhanced by the complexation of lead(II) with various anions (e.g., iodide) [13]. Therefore, by impregnating a membrane with $\mathrm{CPC}$, it is possible to extract lead(II) as its iodide complex (i.e., $\mathrm{Pbl}_{4}{ }^{2-}$ ) onto the membrane. Once adsorbed on the membrane, passing a solution of PAR (pH 8-10) through the disk will form the neutral lead(II): $\mathrm{PAR}_{2}$ complex, and transform the yellow-colored PAR to its red-colored lead(II) complex. Equations 3-5 summarizes the overall reaction scheme, noting that the materials confined in the membrane are subscripted with "mem".

$$
\begin{aligned}
& \mathrm{Pb}^{2+}+4 \mathrm{I}^{-} \leftrightarrow \mathrm{Pbl}_{4}{ }^{2-} \\
& \mathrm{Pbl}_{4}^{2-}+\mathrm{CP}^{+} \mathrm{Cl}_{\text {mem }}^{-} \leftrightarrow\left[\mathrm{CP}: \mathrm{Pbl}_{4}\right]_{\text {mem }}{ }^{-}+\mathrm{Cl}^{-} \\
& {\left[\mathrm{CP}: \mathrm{Pbl}_{4}\right]_{\text {mem }}^{-}+2 \mathrm{PAR} \leftrightarrow(\mathrm{PAR})_{2} \mathrm{~Pb}_{\text {mem }}+\mathrm{CP}^{+} \mathrm{I}_{4}^{4-}}
\end{aligned}
$$

To test the viability of this approach, $1.0 \mathrm{~mL}$ of $0.2 \mathrm{M} \mathrm{KI}$ solution was added to an arbitrarily selected volume (20.0 mL) of standard lead(II) solutions (0.03-1.0 ppm), with the resulting mixtures pushed through separate CPC- impregnated membranes. This step was followed by the passaged of $1.0 \mathrm{~mL}$ of $0.05 \%$ PAR solution through each membrane. Treatment with PAR imparted a reddish yellow color to the disk that is diagnostic of the complexation of PAR with lead(II). Workup of the data yielded linear calibration plots of $F(R)$ vs. lead(II) concentration between 0.05 and $1.0 \mathrm{ppm}$.

Based on this success and the need to reduce sample consumption, we next carried out experiments to determine the minimum sample volume needed for an effective analysis. We first reduced the volume by $50 \%$ to $10.0 \mathrm{~mL}$ and repeated the analysis. The calibration curve again showed linearity from 0.03 to $1.0 \mathrm{ppm}$ (Figure 5), with the limit of detection $0.01 \mathrm{ppm}$. Further reduction of the sample volume $(4.0 \mathrm{~mL})$, however, suffered from poor reproducibility, especially at low levels of lead(II) (Figure 6). This situation arises from the large void volume in the sample holder in comparison to the $4.0 \mathrm{~mL}$ sample volume, which resulted in the ineffective passage of the sample through the membrane. We are presently redesigning the sample holder in order to overcome this obstacle, and tests for interferences will be carried out in the near future.

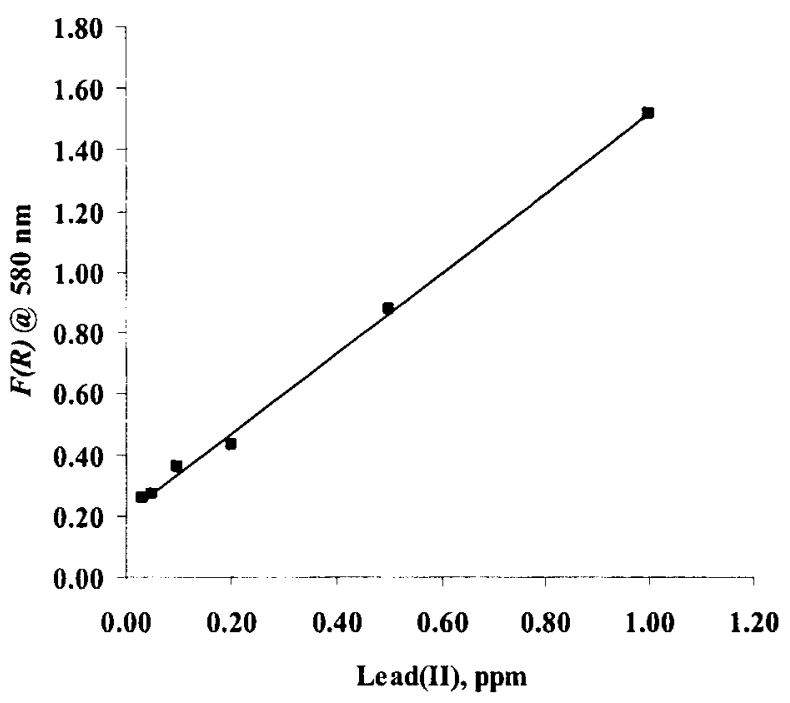

Figure 5. Calibration plot for lead(II) with PAR for $10.0 \mathrm{~mL}$ sample volume.

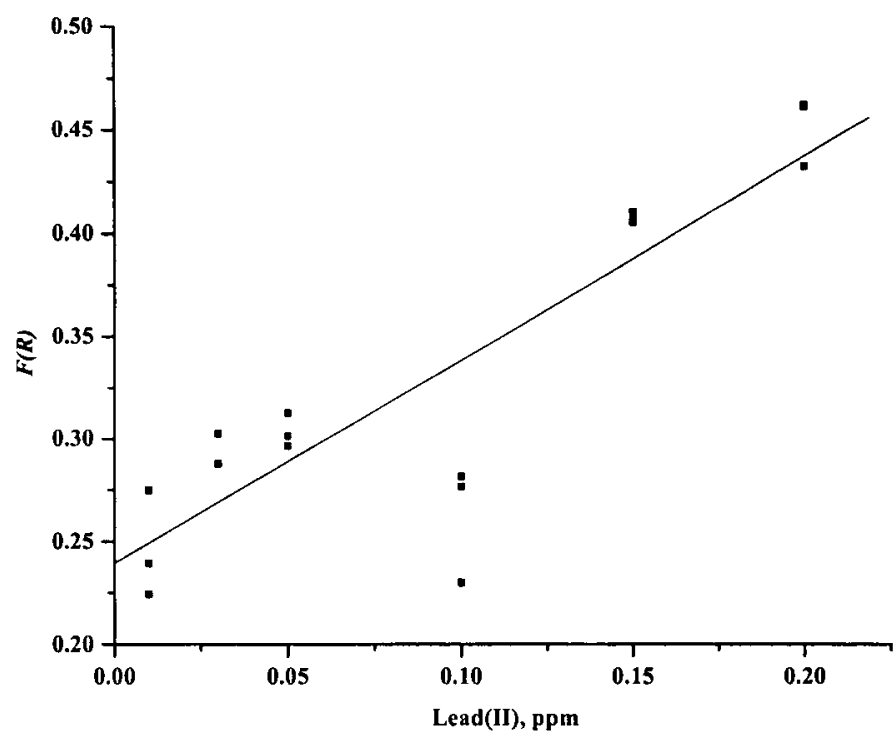

Figure 6. Calibration plot for lead(II) with PAR for $4.0 \mathrm{~mL}$ sample volume.

\section{CONCLUSION}

This report provides an update on the development of methods for the application of C-SPE to the determination of nickel(II) and lead(II). The results show that the method utilizing DMG as a nickel(II) precipitating agent operates effectively and is ready to be tested in $\mathrm{KC}-135$ microgravity simulations. In contrast, much more needs to be done in terms of addressing the interference issues in the determination of lead(II) by its complexation with DMTD, and on further assessing the potential utility of PAR. We are particularly interested in unraveling the 
fundamental of factors which result in the ineffectiveness of tartrate as a masking agent when used in C-SPE.

\section{ACKNOWLEDGMENTS}

This work was supported by NASA contracts NAG91191 and NAG91510, and the Microanalytical Instrumentation Center at lowa State University. The Ames Laboratory is operated by lowa State University under US Department of Energy contract W-7405-eng-82.

\section{REFERENCES}

1. M. P. Arena, M. D. Porter, J. S. Fritz, Anal. Chem. 74 (2002) 185-190.

2. M. P. Arena, M. D. Porter, J. S. Fritz, Anal. Chim Acta 482 (2003) 197-207.

3. P. D. Mudgett, 16 September 2003, personal communication.

4. D. K. Plumlee, 32nd International Conference on Environmental Systems, San Antonio, TX, SAE Technical Paper \#2002-01-2537, 2002

5. D. B. Gazda, J. S. Fritz, M. D. Porter, Anal. Chim Acta 508 (2004) 53-59.

6. L. M. Ponton, D. Gazda, R. J. Lipert, J. S. Fritz, M. D. Porter, J. Rutz, P. Mudgett, D. Dungan, and J. Schultz, 33th International Conference on Environmental Systems, Vancouver, B.C., Canada, SAE Technical Paper \#2003-01-2408, 2003

7. M. Arena, M. Porter, J. Fritz, P. Mudgett, J. Rutz, and J. Shultz, 32th International Conference on Environmental Systems, San Antonio, TX, SAE Technical Paper \#2002-01-2535, 2002.

8. J. S. Fritz, M. P. Arena, S. A. Steiner, M. D. Porter, J. Chromatogr. A 997 (2003) 41-50.

9. D. B. Gazda, R. J. Lipert, J. S. Fritz, M. D. Porter, J. Rutz, P. Mudgett, and J. Shultz, 33th International Conference on Environmental Systems, Vancouver, B.C., Canada, SAE Technical Paper \#2003-01-2406, 2003

10. KC-135 flight report, 2004 , in preparation

11. G. Kortum, Reflectance Spectroscopy-Principles, Methods, Applications, Springer, New York, 1969, pp. 106-116.

12. V. P. Dedkova, O. P. Shvoeva, and S. B. Savvin, J. Anal. Chem. 58 (2003) 230-235.

13. O. P. Shvoeva, V. P. Dedkova, and S. B. Savvin, J. Anal. Chem. 56 (2001) 1080-1083.

14. O. P. Shvoeva, V. P. Dedkova, and S. B. Savvin, J. Anal. Chem. 56 (2001) 376-379.

15. S. L. C. Ferreira, V. L. C. Costa, V de B Pontes, A. C. Spinda Costa, I. Quim, Quimica Analitica (Barcelona) 15 (1996) 154-156.

16. M. J. Ahmed, M. Al Mamun, Talanta 55 (2001) 4354.
17. Z. Marczenko, Spectrophotometric Determination of Elements, John Wiley \& Sons Inc., New York, 1976.

18. R. P. Paradkar, R. R. Williams, Anal. Chem. 66 (1994) 2752-2756.

19. A. P. Argekar, A. K. Shetty, Talanta 45 (1998) 909915.

20. A. I. Busev, V. G. Tipëtisova, V. M. Ivanov, Handbook of the analytical chemistry of rare elements, Ann Arbor-Humphrey Science Publishers, Ann Arbor, MI, 1970. 